\title{
B. KAHN
}

\section{Erratum. Quelques remarques sur le $u$-invariant}

Séminaire de Théorie des Nombres de Bordeaux, tome 3, no 1 (1991), p. 247

<http://www.numdam.org/item?id=JTNB_1991_3_1_247_0>

(C) Université Bordeaux 1, 1991, tous droits réservés.

L'accès aux archives de la revue "Séminaire de Théorie des Nombres de Bordeaux » (http://jtnb.cedram.org/) implique l'accord avec les conditions générales d'utilisation (http://www.numdam.org/conditions). Toute utilisation commerciale ou impression systématique est constitutive d'une infraction pénale. Toute copie ou impression de ce fichier doit contenir la présente mention de copyright.

\section{Numdam}

Article numérisé dans le cadre du programme

Numérisation de documents anciens mathématiques

http://www.numdam.org/ 
Séminaire de Théorie des Nombres, Bordeaux 3 (1991), 247

\title{
Erratum. \\ Quelques remarques \\ sur le $u$-invariant
}

\author{
par B. KAHN
}

Pasquale Mammone et Jean-Pierre Tignol m'ont signalé une lacune dans la démonstration de [1], proposition 2,3) : à la première ligne de la page 159 , l'algèbre $\mathbf{A}$ pourrait ne pas être produit tensoriel de 3 algèbres de quaternions ; $A \otimes \kappa_{5}$ pourrait donc être à division. Par conséquent, l'énoncé de loc. cit. est peut-être incorrect.

Cette erreur se présente aussi au paragraphe suivant : l'algèbre générique d'exposant 2 et de degré $2^{m}$ n'est pas produit tensoriel d'algèbres de quaternions dès que $m \geq 3$.

Pour clarifier la situation, il faut introduire un troisième invariant $\lambda^{\prime \prime}(F)$ :

$\lambda^{\prime \prime}(F)=\sup \left\{\lambda^{\prime \prime} \mid\right.$ il existe un corps gauche de centre $F$, d'exposant 2 et de degré $2^{\lambda^{\prime \prime}}$.

Il est évident que pour tout corps $\mathrm{F}$ on a $\lambda^{\prime}(F) \leq \lambda^{\prime \prime}(F) \leq \lambda(F)$. De plus, les arguments de [1] démontrent en fait le résultat suivant :

Proposition. 1) Il existe une fonction $f$ telle que, pour tout corps $F$, on ait

$\lambda(F) \leq f\left(\lambda^{\prime \prime}(F)\right)$.

2) $S i \lambda^{\prime \prime}(F)=3$, on a $\lambda(F)=3$ ou 4 .

Il semble possible qu'on puisse choisir $f(n)=2^{n-1}$. J'ignore s'il existe un corps $\mathrm{F}$ tel que $\lambda^{\prime}(F)$ soit fini et $\lambda(F)$ infini. D'après [1], prop. 2 2) et th. 3 a), il faut pour cela que $\lambda^{\prime}(F) \geq 3$ et $c d_{2}(F) \geq 3$.

\section{RÉFÉRENCE}

[1] B. KAHN, Quelques remarques sur le u-invariant, Sém. Th. des Nombres Bordeaux 2 (1990), 155-161. 